\title{
Validación del contenido de un guion de entrevista sobre la competencia digital docente en Educación Superior
}

\author{
Angelina Lorelí Padilla-Hernández ${ }^{1}$, Vanesa M. ${ }^{a}$ Gámiz-Sánchez ${ }^{2}$ M. ${ }^{a}$ Asunción \\ Romero-López ${ }^{2}$
}

angelina.loreli@gmail.com, vanesa@ugr.es, romerol@ugr.es

${ }^{1}$ Becaria CONACYT-México y doctoranda en la Universidad de Granada, Campus Cartuja s/n, 18011, Granada, España.

${ }^{2}$ Universidad de Granada, Facultad de Ciencias de la Educación, Campus Cartuja s/n, 18011, Granada, España.

DOI: $10.17013 /$ risti.32.1-16

\begin{abstract}
Resumen: Este artículo presenta un estudio para obtener evidencias de validez del contenido de un guion de entrevista en profundidad, en el marco de una investigación sobre el desarrollo de la competencia digital de profesores universitarios. La técnica utilizada fue la evaluación por juicio de expertos, que se centró en los criterios: claridad, pertinencia e importancia, y contó con la participación de docentes investigadores de México, España y Chile. La revisión integradora de la evaluación incluyó el análisis cuantitativo de las calificaciones de los jueces, mediante el Índice de Validez de Contenido y los estadísticos Kappa de Cohen y Kappa de Fleiss, así como el análisis de contenido de los comentarios. Como resultado se obtuvo una versión acotada del guion de entrevista, incluido como anexo. Además, se identificaron aspectos en debate sobre la conceptualización de esta competencia docente en la Educación Superior.
\end{abstract}

Palabras-clave: Competencia digital; Docente; Educación Superior; Validación; Entrevista.

\section{Content validity study of an interview guide about teachers' digital competence in Higher Education}

\begin{abstract}
This paper presents a study to obtain content validity evidence of an in-depth interview guide, in the context of a research about the development of digital competence in Higher Education teachers. The technique used was the expert judgment panel, focused on the following criteria: clarity, relevance and importance. Professors from Mexico, Spain and Chile participated as experts. The integrative review of the evaluation included statistical analysis, using the Content Validity Index and the Kappa of Cohen and Kappa de Fleiss indexes, as well as the content analysis of the comments made by the judges. As a result, a more focused version
\end{abstract}


of the interview guide was obtained, included as an annex. Furthermore, aspects in debate about the conceptualization of this teachers' competence were compiled.

Keywords: Digital competence; Teacher; Higher Education; Validity; Interview.

\section{Introducción}

La participación del profesorado universitario es fundamental para enfrentar el desafío de la integración crítica de las Tecnologías de Información y Comunicación (TIC) en la Educación Superior. Los docentes, con su conocimiento, experiencia y visión, toman decisiones sobre la manera de aprovechar las posibilidades y enfrentar las limitaciones de prácticas y recursos digitales para apoyar el aprendizaje de los estudiantes y su propio desarrollo profesional. De hecho, el rol docente ha sido uno de los temas de interés de la Tecnología Educativa en los últimos 20 años (Prendes, 2018).

En esta línea, el concepto de competencia digital docente (CDD) ayuda a comprender la competencia profesional del perfil del profesorado universitario, necesaria para la integración adecuada de las TIC (Prendes, Martínez \& Gutiérrez, 2018; Tejada \& Pozos, 2018; Zabalza, 2003). En el contexto actual se espera que los docentes aprovechen una amplia gama de herramientas digitales con propósitos de innovación y de mejora educativa (Adams Becker et al., 2017; UNESCO, 2011). Sin embargo, el foco principal de la CDD no está en la tecnología, sino en las competencias movilizadas y las decisiones reflexivas que los profesores toman para incorporar las tecnologías digitales de manera estratégica y pedagógica (Pozos, 2013) y su influencia en la competencia digital de los estudiantes (Redecker, 2017).

Aunque existen avances en la conceptualización de la CDD, resulta complejo delimitar su significado. La propia definición de competencia digital es plural, multifacética y contextual (Ala-Mutka, 2011; Gallardo-Echenique, Minelli, Marqués-Molias \& EsteveMon, 2015; Janssen et al., 2013). Además, se vincula a la influencia del ámbito familiar, al capital cultural y al logro de la inclusión digital (Martínez-Piñeiro, Vila \& Grewerc, 2018). Por otra parte, es necesario considerar los rasgos específicos de la docencia universitaria, como los ejes de actuación profesional (docencia, investigación, gestión, transferencia de conocimiento), los enfoques metodológicos y didácticos, el campo disciplinar, las estrategias y recursos digitales empleados, y los contextos institucionales y sociales que podrían variar los atributos de esta competencia. En el contexto iberoamericano existen propuestas para caracterizar la CDD del profesorado universitario, por ejemplo, la síntesis de Carrera \& Coiduras (2012), el perfil propuesto por Rangel (2016), los modelos de Prendes et al. (2018) y Tejada \& Pozos (2018), así como numerosos estudios e instrumentos para su diagnóstico, evaluación y certificación.

En general, la revisión de literatura muestra una tendencia deductiva y cuantitativa para evaluar la CDD, es decir, a partir del uso de cuestionarios, encuestas y declaraciones de desempeños. Aún son pocas las aproximaciones cualitativas y enfocadas en el carácter dinámico de la CDD. Si partimos de la comprensión de competencia desde el enfoque socioconstructivista, sería necesario reconocer su carácter procesual y cualitativo (DíazBarriga, 2011). Por lo tanto, existe la oportunidad de contribuir en esta línea al considerar el carácter evolutivo de la CDD como foco principal de estudio, anclado en el desarrollo profesional docente. 
La investigación más amplia a la que pertenece este estudio tiene como propósito caracterizar la adquisición y el desarrollo en la práctica de la CDD de docentes universitarios de México y España, desde una perspectiva cualitativa y mediante el enfoque biográfico narrativo. Como parte del diseño metodológico surgió la necesidad de revisar el diseño del instrumento principal: una entrevista en profundidad de tipo biográfico, organizada en varias sesiones (Fernández, 2006; Kelchtermans, 1999). Se requería que esta mantuviera un carácter abierto y a la vez común entre los relatos de vida de los participantes, a partir de líneas cronológicas y temáticas (Bolívar, Domingo \& Fernández, 2001). De la revisión de literatura especializada se obtuvo un listado amplio de dimensiones, categorías e indicadores, por lo que fue necesario realizar un estudio de obtención de evidencias de validez de contenido.

En síntesis, la validez se entiende como el grado en que la teoría y la evidencia son un sustento para la interpretación de los datos que se obtienen mediante un instrumento, en concordancia con los objetivos del mismo (Bandalos, 2018; Pedrosa, Suárez-Álvarez \& García-Cueto, 2013). Según Cabero \& Llorente (2013) el juicio de expertos se utiliza en el ámbito de la educación y las tecnologías como técnica para evaluar instrumentos, recursos educativos y propuestas de aprendizaje, por ejemplo, para revisar su adecuación a la población objetivo (Rodrigues, Martins, Pinto \& Teixeira, 2018).

Además, el juicio de expertos tiene la ventaja de generar información detallada acerca de “(...) contenidos y temáticas difíciles, complejas y novedosas o poco estudiadas" (Cabero \& Llorente, 2013: 14), como es el caso de la CDD en términos de su evolución. Algunos estudios sobre la CDD de profesores universitarios han utilizado esta técnica para evaluar los instrumentos de investigación empleados (Cejas-León \& Navío, 2016; Rangel, 2016). A continuación, se comparte el método seguido para la realización de este estudio.

\section{Método}

El juicio de expertos se considera una fuente de evidencias de validez del contenido puesto que las evaluaciones de los jueces ayudan a revisar la relevancia y representatividad de los ítems, así como su relación con los propósitos del instrumento y con el dominio de interés (Escobar-Pérez \& Cuervo-Martínez, 2008; Pedrosa et al., 2013; Sireci, 1998). Forma parte de la búsqueda continua de la validez y del rigor en la investigación (Bandalos, 2018; Hernández-Sampieri, Fernández \& Baptista, 2014). En otras palabras, esta técnica proporciona una base para la toma de decisiones a fin de mejorar el diseño de los instrumentos de investigación.

El objetivo del presente estudio fue obtener evidencias de la validez de contenido de las dimensiones e indicadores sobre la evolución de la competencia digital docente del profesorado universitario, mediante la evaluación por juicio de expertos, a fin de mejorar el guion de entrevista en profundidad. Los objetivos específicos fueron:

- Valorar la pertinencia de las dimensiones e indicadores para caracterizar la evolución de la CDD del docente universitario.

- Identificar aspectos por añadir o eliminar para cubrir el dominio de interés.

- Recopilar observaciones para la mejora del instrumento. 
En cuanto al desarrollo del estudio, este se organizó en tres fases: preparatoria, desarrollo de la evaluación y toma de decisiones. Como parte de la fase preparatoria, se seleccionaron los temas para el guion de entrevista de acuerdo a la revisión de literatura especializada (estudios del ámbito e instrumentos de investigación) y su análisis descriptivo (Padilla-Hernández, Gámiz-Sánchez \& Romero-López, 2018). Las cuatro dimensiones identificadas se desglosaron en 17 categorías y 65 indicadores que representan los temas sugeridos para la entrevista.

Se construyeron los criterios de evaluación con sus respectivos límites de aceptación y revisión a partir del cálculo de medidas de dispersión (ver tabla 1). Para el criterio de pertinencia se utilizó el Índice de Validez de Contenido (IVC) (Lawshe, 1975) para valorar el grado de acuerdo o consenso entre los expertos sobre la adecuación de los ítems y de las dimensiones del instrumento (Delgado-Rico, Carretero-Dios \& Ruch, 2012; Pedrosa et al., 2013; Polit, Beck \& Owen, 2007).

\begin{tabular}{|c|c|c|c|}
\hline Criterio & Descripción & Escala & $\begin{array}{l}\text { Límite para su revisión/ } \\
\text { aceptación }\end{array}$ \\
\hline Claridad & $\begin{array}{l}\text { La redacción del ítem es clara y } \\
\text { su significado se comprende sin } \\
\text { ambigüedad. }\end{array}$ & $\begin{array}{l}\text { Dicotómica: } \\
\text { Sí, No. }\end{array}$ & $\begin{array}{l}\text { Porcentaje de respuestas } \\
\text { afirmativas: 80\% (Escobar-Pérez \& } \\
\text { Cuervo-Martínez, 2008). }\end{array}$ \\
\hline Pertinencia & $\begin{array}{l}\text { El indicador es adecuado para el } \\
\text { objetivo del estudio. }\end{array}$ & $\begin{array}{l}\text { Dicotómica: } \\
\text { Sí, No. }\end{array}$ & $\begin{array}{l}\text { Promedio del IVC por dimensión: } \\
\text { o.8o (Delgado-Rico et al., 2012). } \\
\text { IVC de los indicadores: 0.67 (Polit } \\
\text { et al., 2007). }\end{array}$ \\
\hline Importancia & $\begin{array}{l}\text { Grado de importancia y } \\
\text { significatividad del indicador } \\
\text { para la dimensión. }\end{array}$ & $\begin{array}{l}\text { Tipo Likert: } \\
\text { 1: Muy poca } \\
\text { 2: Poca } \\
\text { 3: Bastante } \\
\text { 4: Mucha }\end{array}$ & $\begin{array}{l}\text { Media (M): menos de } 3 . \\
\text { Desviación estándar (DE) como } \\
\text { información adicional. }\end{array}$ \\
\hline
\end{tabular}

Tabla 1 - Criterios de evaluación

Como el IVC no excluye la posibilidad de acuerdo debida al azar se recurrió a medidas complementarias de estimación de la consistencia entre las evaluaciones, como el índice Kappa de Cohen (porcentaje de acuerdo entre pares y promedio de pares) y el Kappa de Fleiss, específico para múltiples evaluadores (Delgado-Rico et al., 2012; Wynd, Schmidt \& Atkins, 2003). Estos índices se emplearon para la revisión general de las dimensiones del instrumento. El límite para un acuerdo moderado y aceptable fue de 0.41 para el promedio del Kappa de Cohen y para el resultado del índice Kappa de Fleiss (DelgadoRico et al., 2012; López de Ullibarri \& Pita, 1999).

Se diseñó una planilla como instrumento para la evaluación, con los criterios de evaluación y con espacio para los comentarios de los jueces. Además, se elaboró una carta de invitación con el contexto de la investigación.

Seleccionamos a los profesores e investigadores participantes según el procedimiento del biograma (Cabero \& Llorente, 2013), de acuerdo a la revisión de su trayectoria profesional. Los criterios de inclusión fueron: conocimiento y experiencia investigadora 
en el tema y el enfoque metodológico a fin de obtener una retroalimentación pertinente para el instrumento (Davis, 1992), amplia experiencia como docentes universitarios por la empatía con el perfil y la experiencia profesional (Hynes, Richard \& Kubany, 1995), Iberoamérica como ubicación geográfica debido al contexto del estudio, equidad de género y disposición para participar. A partir de estos criterios se construyó una muestra intencional de especialistas.

Seis profesores investigadores aceptaron la invitación, cada uno de ellos reúne más de 20 años de trayectoria profesional en la docencia universitaria y son expertos en las líneas de investigación (Tecnología educativa, Aprendizaje en red, Competencia digital, Desarrollo profesional docente) o en el enfoque biográfico-narrativo. Una de las profesoras reside en Chile (Profesora A), dos docentes en México (Profesor B y Profesora E) y tres en España (Profesora D, Profesores C y F).

En la fase de desarrollo se llevó a cabo el contacto con los docentes investigadores y la recogida de datos. Los materiales se recopilaron vía correo electrónico de forma individual, aunque también se desarrollaron conversaciones personales para profundizar en algunos aspectos de las sugerencias. Las calificaciones se registraron en una hoja de datos. Los cálculos de las medidas de dispersión y del IVC fueron realizados con apoyo de fórmulas en la hoja de datos. Para el cálculo de los índices Kappa se utilizó la herramienta en línea ReCal3 (Freelon, 2010, 2017), cuyos resultados se verificaron con el programa IBM SPSS Statistics (versión 24.0).

La fase de toma de decisiones sobre los cambios al instrumento se basó en el análisis cualitativo de las calificaciones, según los límites expresados en la tabla 1, y en la interpretación cualitativa de las observaciones. Como sugieren Polit et al. (2007) se identificaron los ítems que requerían revisión o descarte, para luego contrastar esta revisión con los comentarios de los jueces. La valoración cualitativa de los comentarios se realizó mediante el análisis de contenido. Las aportaciones se codificaron según los temas de la entrevista como categorías previamente establecidas (Bardin, 1996). Una categoría nueva emergió del conjunto de comentarios: recomendaciones generales para la investigación. Finalmente, una vez que el guion fue corregido según los resultados de la evaluación del juicio de expertos, se desarrolló un ejercicio piloto de entrevista con dos docentes.

\section{Resultados}

\section{1. Índices generales por dimensión}

En la tabla 2 se presentan los índices obtenidos sobre la concordancia entre evaluadores y el promedio de IVC por dimensión.

En términos generales, los índices de concordancia manifestaron un acuerdo moderado entre los evaluadores, no existiendo evidencias empíricas de inconsistencia entre las evaluaciones de los jueces. El promedio del IVC de las dos últimas dimensiones se presentó ligeramente por debajo del o.80, por lo tanto, fue necesario revisar su construcción. A continuación, se detalla la evaluación para cada dimensión y sus indicadores. 


\begin{tabular}{lllll}
\hline Dimensión & $\begin{array}{l}\text { Pertinencia, } \\
\text { promedio del } \\
\text { IVC }\end{array}$ & $\begin{array}{l}\text { Porcentaje de } \\
\text { acuerdo por pares } \\
\text { Kappa de Cohen }\end{array}$ & $\begin{array}{l}\text { Promedio del } \\
\text { Kappa de Cohen } \\
\text { por pares }\end{array}$ & $\begin{array}{l}\text { Índice Kappa } \\
\text { de Fleiss }\end{array}$ \\
\hline $\begin{array}{l}\text { Desarrollo profesional } \\
\text { docente }\end{array}$ & 0.81 & $68.64 \%$ & 0.497 & 0.473 \\
\hline $\begin{array}{l}\text { Evolución de la CDD } \\
\text { Áreas de la CDD }\end{array}$ & 0.84 & $75.29 \%$ & 0.574 & 0.563 \\
\hline $\begin{array}{l}\text { Valoración crítica } \\
\text { del uso de TIC en } \\
\text { Educacion Superior }\end{array}$ & 0.75 & $75.29 \%$ & 0.566 & 0.553 \\
\hline $\begin{array}{l}\text { Promedio general } \\
\text { Tromed }\end{array}$ & 0.78 & $68.33 \%$ & 0.508 & 0.473 \\
\hline
\end{tabular}

Tabla 2 - Índices por dimensión y promedio general

\subsection{Desarrollo profesional docente}

En esta dimensión (D1), el criterio de pertinencia tuvo un mayor grado de concordancia entre evaluadores, con un porcentaje de acuerdo entre pares de un $84.44 \%$, en contraste con la claridad (67.4\%) y la importancia de sus ítems (54.07\%). La tabla 3 muestra las puntuaciones de los indicadores de la D1:

\begin{tabular}{|c|c|c|c|c|c|}
\hline \multirow[t]{2}{*}{ Categoría } & \multirow[t]{2}{*}{ Indicador } & \multirow{2}{*}{$\begin{array}{l}\text { Claridad } \\
\text { (\%Sí) }\end{array}$} & \multirow{2}{*}{$\begin{array}{l}\text { Pertinencia } \\
\text { (IVC) }\end{array}$} & \multicolumn{2}{|c|}{ Importancia } \\
\hline & & & & (M) & (DE) \\
\hline \multirow[t]{3}{*}{$\begin{array}{l}\text { Trayectoria } \\
\text { profesional }\end{array}$} & $\begin{array}{l}\text { Razones para elegir la docencia } \\
\text { como profesión }\end{array}$ & 100 & 1 & $3 \cdot 3$ & 0.82 \\
\hline & Evolución de la carrera profesional & $83 \cdot 3$ & 1 & 3.7 & 0.52 \\
\hline & Incidentes críticos & $50^{*}$ & $0.33^{*}$ & $2.7^{*}$ & o \\
\hline \multirow[t]{3}{*}{$\begin{array}{l}\text { Perfil } \\
\text { profesional }\end{array}$} & $\begin{array}{l}\text { Autopercepción y motivaciones } \\
\text { profesionales }\end{array}$ & 100 & 1 & 4 & 0 \\
\hline & $\begin{array}{l}\text { Rasgos del conocimiento pedagógico } \\
\text { y disciplinar }\end{array}$ & $83 \cdot 3$ & $0.67^{*}$ & 3.7 & 0.52 \\
\hline & Entorno socio-profesional & $83 \cdot 3$ & 1 & 3.8 & 0.41 \\
\hline \multirow{3}{*}{$\begin{array}{l}\text { Perspectiva de } \\
\text { futuro }\end{array}$} & Valoración del momento presente & $66.7^{*}$ & $0.33^{*}$ & 3 & 1.26 \\
\hline & Metas de desarrollo profesional & 83.3 & 1 & 3.8 & 0.41 \\
\hline & $\begin{array}{l}\text { Prospectiva de la universidad, la } \\
\text { sociedad y las TIC }\end{array}$ & 83.3 & 1 & 3.8 & 0.41 \\
\hline
\end{tabular}

Nota: Con asterisco $\left({ }^{*}\right)$ se marcan los resultados de los ítems a revisar.

Tabla 3 - Cálculos e IVC para los indicadores de la D1: Desarrollo profesional docente

Se eliminaron los ítems que obtuvieron un IVC bajo por su ambigüedad o carácter analítico, según las observaciones de los jueces. Los temas agrupados en las categorías "Perfil profesional" y "Perspectiva de futuro" se reorganizaron para evitar su solapamiento con otros ítems. 
Se añadieron temas relacionados con: “(...) experiencias formativas tempranas: antecedentes relacionados con la docencia; antecedentes relacionados con las TIC; experiencia en la Educación Secundaria y Preparatoria en relación con las TIC”, según lo sugirió el Profesor F. Otra observación de los jueces fue destacar el carácter reflexivo de la docencia dado que resulta transversal en el ejercicio de relatar las experiencias relacionadas con la CDD.

\subsection{Evolución de la competencia digital docente}

En esta dimensión (D2), los promedios de acuerdo entre pares de los criterios claridad y pertinencia presentaron una consistencia alta (81.24\% y 86.28\% respectivamente), mientras que el de importancia obtuvo un porcentaje del 57.65\%. La tabla 4 presenta la calificación de los indicadores de la D2.

\begin{tabular}{|c|c|c|c|c|c|}
\hline \multirow[t]{2}{*}{ Categoría } & \multirow[t]{2}{*}{ Indicador } & \multirow{2}{*}{$\begin{array}{l}\text { Claridad } \\
\text { (\%Sí) }\end{array}$} & \multirow{2}{*}{$\begin{array}{l}\text { Pertinencia } \\
\text { (IVC) }\end{array}$} & \multicolumn{2}{|c|}{ Importancia } \\
\hline & & & & (M) & (DE) \\
\hline \multirow[t]{4}{*}{$\begin{array}{l}\text { Desarrollo de la } \\
\text { CDD }\end{array}$} & $\begin{array}{l}\text { Primeras experiencias para la } \\
\text { adquisición de la CDD }\end{array}$ & 100 & 1 & 4 & o \\
\hline & Hitos para el desarrollo de la CDD & $66.7^{*}$ & 1 & 4 & o \\
\hline & $\begin{array}{l}\text { Dificultades en relación con la } \\
\text { competencia digital }\end{array}$ & 100 & 1 & 4 & o \\
\hline & $\begin{array}{l}\text { Influencia de otros ámbitos } \\
\text { (familiar, social, laboral) }\end{array}$ & 100 & 1 & $3 \cdot 7$ & 0.52 \\
\hline \multirow{3}{*}{$\begin{array}{l}\text { Uso de TIC } \\
\text { en los ejes de } \\
\text { la docencia } \\
\text { universitaria }\end{array}$} & Docencia & 100 & 1 & 3.8 & 0.41 \\
\hline & Investigación & 100 & 1 & 3.5 & 0.84 \\
\hline & Gestión & 100 & 1 & 3.5 & 0.84 \\
\hline \multirow{4}{*}{$\begin{array}{l}\text { Aprendizaje } \\
\text { continuo }\end{array}$} & Exploración sobre educación y TIC & $50^{*}$ & $0.67^{*}$ & $3 \cdot 7$ & 0.52 \\
\hline & Formación continua & 100 & 1 & 3.7 & 0.52 \\
\hline & $\begin{array}{l}\text { Colaboración (docentes, } \\
\text { estudiantes, comunidad) }\end{array}$ & $83 \cdot 3$ & 1 & 3.8 & 0.41 \\
\hline & $\begin{array}{l}\text { Proyectos de investigación o de } \\
\text { innovación }\end{array}$ & 100 & 1 & $3 \cdot 3$ & 0.82 \\
\hline \multirow{4}{*}{$\begin{array}{l}\text { Rutinas, } \\
\text { espacios } \\
\text { de trabajo } \\
\text { y recursos } \\
\text { digitales }\end{array}$} & $\begin{array}{l}\text { Actividades y uso del tiempo en la } \\
\text { jornada laboral }\end{array}$ & $83 \cdot 3$ & $0.67^{*}$ & $3 \cdot 3$ & 0.82 \\
\hline & Espacios de trabajo & 100 & $0.67^{*}$ & 3.5 & 0.84 \\
\hline & Recursos digitales & 100 & 1 & 4 & $\mathrm{o}$ \\
\hline & $\begin{array}{l}\text { Emociones de las experiencias } \\
\text { en red }\end{array}$ & $83 \cdot 3$ & 1 & 3.8 & 0.41 \\
\hline \multirow{2}{*}{$\begin{array}{l}\text { Uso de TIC en la } \\
\text { vida cotidiana }\end{array}$} & Finalidades & $50^{*}$ & $0.00^{*}$ & $2.7^{*}$ & 1.3 \\
\hline & $\begin{array}{l}\text { Relación con su competencia } \\
\text { digital docente }\end{array}$ & $83 \cdot 3$ & $0.33^{*}$ & 3.7 & 0.52 \\
\hline
\end{tabular}

Nota: Con asterisco $\left(^{*}\right)$ se marcan los resultados de los ítems a revisar.

Tabla 4 - Cálculos e IVC para los indicadores de la D2: Evolución de la CDD 
Esta dimensión presentó varios puntos a revisar. Por un lado, fueron eliminados los ítems que obtuvieron un IVC bajo debido a la falta de claridad, ambigüedad o amplitud. Como ejemplo está la categoría "Uso de TIC en la vida cotidiana" que fue eliminada porque el foco de la investigación es en la CDD y los relatos de los docentes podrían expresar la influencia de otros ámbitos en el desarrollo de esta competencia.

Los jueces sugirieron añadir la vinculación o transferencia de conocimiento a los ejes de la docencia universitaria como parte del trabajo académico. También se incluyeron los proyectos de innovación y de investigación relacionados con el aprovechamiento de las TIC. Se renombraron ciertos ítems para hacerlos más claros y diferenciados, como el caso de "Exploración sobre educación y TIC" renombrado como: "Estrategias para actualizar su CDD”.

\section{4.Áreas de la competencia digital docente}

La tercera dimensión (D3) mostró el mayor porcentaje de acuerdo entre pares en cuanto a la claridad (81.24\%), mantuvo un buen nivel de concordancia en el criterio de pertinencia (73.89\%) y presentó el grado más alto de acuerdo sobre el criterio de importancia (63.7\%). La tabla 5 reúne las calificaciones de los ítems de la D3.

El panel de expertos señaló ítems cuya formulación resultó menos clara para abordar la CDD. La categoría "Pedagogía” recibió una crítica debido a su enfoque, que según varios jueces resultaba poco actual, tradicional o difícil de abordar. Algunos ítems fueron eliminados por su formulación poco clara, entre ellos la "Generación de conocimiento" y “Adecuación de recursos digitales". También se descartaron los siguientes indicadores por su bajo IVC y por las observaciones de los jueces: "Netiqueta", "Programación" y "Participación ciudadana". Por ejemplo, el profesor C señaló: “(...) la "Programación” no debe contemplarse como objetivo (y por tanto pongo en cuestión el área) en la CDD.”

Varios jueces coincidieron en el poco nivel de concreción de la categoría "Ética, salud y seguridad", como detalla la profesora D: "Aprecio cierto solapamiento [entre varias] cuestiones de este mismo apartado (...)”. Así, revisamos de nuevo la literatura e identificamos dos ejes generales: "Implicaciones éticas y uso responsable de tecnologías digitales", y "Salud, seguridad y prevención de riesgos". Éstos se incluyeron como indicadores de una nueva categoría: "Reflexiones sobre su CDD”.

Finalmente, esta dimensión se transformó en una categoría de la D2 con el propósito de acotar el acercamiento al problema de investigación. En este cambio las áreas de la CDD pasaron a ser indicadores, dotando a los ítems de un carácter más inductivo y abierto al contenido de los relatos de los docentes. En el caso del área pedagógica, se valorarán los aspectos concretos que surjan en la conversación con los docentes.

\subsection{Valoración crítica del uso de TIC en Educación Superior}

En términos generales esta dimensión (D4) reunió el menor porcentaje de acuerdo entre pares, sobre todo en el criterio de importancia (55.56\%). Los porcentajes de la pertinencia (80.21\%) y la claridad $(78.84 \%)$ presentaron una diferencia menor. En la tabla 6 se encuentran las puntuaciones de los ítems de la D4. 


\begin{tabular}{|c|c|c|c|c|c|}
\hline \multirow[t]{2}{*}{ Categoría } & \multirow[t]{2}{*}{ Indicador } & \multirow{2}{*}{$\begin{array}{l}\text { Claridad } \\
\text { (\%Sí) }\end{array}$} & \multirow{2}{*}{$\begin{array}{l}\text { Pertinencia } \\
\text { (IVC) }\end{array}$} & \multicolumn{2}{|c|}{ Importancia } \\
\hline & & & & (M) & (DE) \\
\hline \multirow[t]{4}{*}{ Pedagógica } & $\begin{array}{l}\text { Planificación de experiencias de } \\
\text { aprendizaje }\end{array}$ & 100 & $0.33^{*}$ & 3 & 0.55 \\
\hline & $\begin{array}{l}\text { Desarrollo de experiencias de } \\
\text { aprendizaje }\end{array}$ & 100 & $0.67^{*}$ & $3 \cdot 3$ & o \\
\hline & Evaluación & 100 & $0.67^{*}$ & 3.2 & 0.45 \\
\hline & $\begin{array}{l}\text { Valoración del potencial del uso de } \\
\text { TIC en educación }\end{array}$ & $83 \cdot 3$ & $0.33^{*}$ & $2.3^{*}$ & 0.84 \\
\hline \multirow[t]{4}{*}{ Informacional } & $\begin{array}{l}\text { Fuentes de información y recursos } \\
\text { para la docencia }\end{array}$ & 100 & 1 & 4 & o \\
\hline & $\begin{array}{l}\text { Búsqueda y selección de } \\
\text { información }\end{array}$ & 100 & 1 & 4 & o \\
\hline & $\begin{array}{l}\text { Organización y recuperación de } \\
\text { información }\end{array}$ & 100 & 1 & 4 & o \\
\hline & Generación de conocimiento & $66.7^{*}$ & $0.67^{*}$ & $3 \cdot 3$ & o \\
\hline \multirow{5}{*}{$\begin{array}{l}\text { Comunicativo- } \\
\text { colaborativa }\end{array}$} & Comunicación en red & 100 & 1 & $3 \cdot 3$ & $\mathrm{O}$ \\
\hline & Colaboración en red & 100 & 1 & $3 \cdot 3$ & o \\
\hline & Netiqueta & $83 \cdot 3$ & $0.33^{*}$ & $2.8^{*}$ & 0.98 \\
\hline & Participación ciudadana & $83 \cdot 3$ & $0.00^{*}$ & $3 \cdot 5$ & 0.84 \\
\hline & Gestión de la identidad digital & $83 \cdot 3$ & $0.67^{*}$ & 3.8 & 0.41 \\
\hline \multirow[t]{4}{*}{$\begin{array}{l}\text { Creación de } \\
\text { contenido digital }\end{array}$} & $\begin{array}{l}\text { Creación de material digital } \\
\text { (propio, integración) }\end{array}$ & 100 & 1 & 4 & $\mathrm{O}$ \\
\hline & $\begin{array}{l}\text { Uso de licencias y derechos de } \\
\text { autor }\end{array}$ & $83 \cdot 3$ & $0.67^{*}$ & 3.5 & 0.84 \\
\hline & Adecuación de recursos digitales & $83 \cdot 3$ & $0.67^{*}$ & $3 \cdot 3$ & o \\
\hline & Programación & 100 & $0.33^{*}$ & $3 \cdot 3$ & 1.03 \\
\hline \multirow{6}{*}{$\begin{array}{l}\text { Ética, saludy } \\
\text { seguridad }\end{array}$} & Valores en las prácticas digitales & $83 \cdot 3$ & $0.67^{*}$ & $3 \cdot 3$ & 0 \\
\hline & Equidad de acceso y uso de TIC & $83 \cdot 3$ & 1 & 4 & o \\
\hline & $\begin{array}{l}\text { Hábitos saludables y sostenibles de } \\
\text { uso de TIC }\end{array}$ & $83 \cdot 3$ & $0.67^{*}$ & 3 & 0.89 \\
\hline & Protección de datos y dispositivos & 100 & 1 & 3.7 & 0.82 \\
\hline & Protección del medio ambiente & 100 & 1 & $3 \cdot 7$ & 0.82 \\
\hline & Relación entre sociedad y TIC & $66.7^{*}$ & 1 & 3.5 & 0.55 \\
\hline \multirow[t]{4}{*}{ Técnica } & $\begin{array}{l}\text { Comprensión a nivel usuario de } \\
\text { software, hardware y dispositivos } \\
\text { externos }\end{array}$ & $83 \cdot 3$ & 1 & 4 & $\mathrm{O}$ \\
\hline & $\begin{array}{l}\text { Comprensión de las funciones y la } \\
\text { estructura de Internet }\end{array}$ & $83 \cdot 3$ & 1 & 4 & o \\
\hline & $\begin{array}{l}\text { Identificación de la infraestructura } \\
\text { de los recursos digitales }\end{array}$ & $83 \cdot 3$ & $0.67^{*}$ & $3 \cdot 3$ & o \\
\hline & Resolución de problemas técnicos & 100 & 1 & $3 \cdot 5$ & 0.84 \\
\hline
\end{tabular}

Nota: Con asterisco $\left(^{*}\right)$ se marcan los resultados de los ítems a revisar.

Tabla 5 - Cálculos e IVC para los indicadores de la D3: Áreas de la CDD 


\begin{tabular}{|c|c|c|c|c|c|}
\hline \multirow[t]{2}{*}{ Categoría } & \multirow[t]{2}{*}{ Indicador } & \multirow{2}{*}{$\begin{array}{l}\text { Claridad } \\
\text { (\%Sí) }\end{array}$} & \multirow{2}{*}{$\begin{array}{l}\text { Pertinencia } \\
\text { (IVC) }\end{array}$} & \multicolumn{2}{|c|}{ Importancia } \\
\hline & & & & (M) & (DE) \\
\hline \multirow{5}{*}{$\begin{array}{l}\text { Cambios y } \\
\text { reflexiones en } \\
\text { su docencia }\end{array}$} & Cambios en el perfil docente & $66.7^{*}$ & 1 & 3.7 & 0.52 \\
\hline & Problemáticas del uso de TIC & $66.7^{*}$ & $0.33^{*}$ & 3.2 & 0.45 \\
\hline & $\begin{array}{l}\text { Reflexiones para el uso crítico de } \\
\text { TIC }\end{array}$ & $83 \cdot 3$ & $0.67^{*}$ & $3 \cdot 3$ & o \\
\hline & Vías para compartir su experiencia & $83 \cdot 3$ & 1 & 3.8 & 0.41 \\
\hline & $\begin{array}{l}\text { Necesidades de aprendizaje en } \\
\text { relación con la CDD }\end{array}$ & 100 & 1 & 3.7 & 0.52 \\
\hline \multirow{3}{*}{$\begin{array}{l}\text { La competencia } \\
\text { digital de los } \\
\text { estudiantes }\end{array}$} & Importancia en su formación & 83.3 & $0.67^{*}$ & $3 \cdot 3$ & o \\
\hline & $\begin{array}{l}\text { Desarrollo de la CDD en la } \\
\text { universidad }\end{array}$ & 100 & $0.67^{*}$ & $3 \cdot 3$ & o \\
\hline & Necesidades de aprendizaje & 83.3 & $0.67^{*}$ & $3 \cdot 3$ & o \\
\hline \multirow{4}{*}{$\begin{array}{l}\text { Educación } \\
\text { Superior y } \\
\text { cultura digital }\end{array}$} & $\begin{array}{l}\text { Influencia de la política educativa } \\
\text { en su práctica }\end{array}$ & $66.7^{*}$ & $0.33^{*}$ & 3 & 0.89 \\
\hline & $\begin{array}{l}\text { Percepción de la competencia } \\
\text { digital en su institución }\end{array}$ & 100 & $0.67^{*}$ & 3.7 & 0.52 \\
\hline & $\begin{array}{l}\text { Impacto de la cultura digital en la } \\
\text { Educación Superior y viceversa }\end{array}$ & 100 & 1 & 3.7 & 0.82 \\
\hline & $\begin{array}{l}\text { Desafíos para el uso crítico de TIC } \\
\text { en la Educación Superior }\end{array}$ & 100 & $0.67^{*}$ & 3.8 & 0.41 \\
\hline
\end{tabular}

Nota: Con asterisco $(*)$ se marcan los resultados de los ítems a revisar.

Tabla 6 - Cálculos e IVC para los indicadores de la D4: Valoración crítica del uso de TIC en Educación Superior

Entre las modificaciones a esta dimensión, se destaca la reorganización de la categoría “Cambios y reflexiones sobre su docencia”. Se renombró para distinguir la percepción sobre cambios en la docencia y las reflexiones sobre el desarrollo de la CDD. Una jueza observó la relación directa del contenido de la categoría con la dimensión sobre la evolución de la CDD. Por esto, se añadió una nueva categoría en la segunda dimensión, titulada "Reflexiones sobre su CDD”, que integró nuevos ítems por sugerencia de los jueces: la percepción de beneficios asociados a su CDD y la influencia de las prácticas digitales en la mejora educativa. Cabe destacar el comentario de la profesora D acerca de la conveniencia de tratar: “(...) la influencia de la competencia digital en la mejora de los resultados de aprendizaje o en la mejora de la calidad de la enseñanza universitaria”.

Luego, se amplió la sección dedicada al perfil de los estudiantes o jóvenes universitarios, pues como señaló la profesora E, además del centro en la persona del profesor, sería necesario considerar: “(...) dos elementos que me parecen muy relevantes: (1) los retos para los entornos universitarios; (2) ese otro, el aprendiz”. En cuanto al segundo punto, el profesor B comentó que: “(...) alguna cuestión relacionada con cómo los profesores valoran (o evaden) las competencias digitales de los estudiantes puede ayudar a explicar alguna parte de la evolución del profesor en esas competencias”. Además, se incluyó 
una categoría sobre los grupos docentes donde participa el profesorado por su posible influencia en la CDD.

Fue necesario renombrar esta dimensión como: "Contexto: Educación Superior y cultura digital”, en sintonía con el comentario de la Profesora A sobre la importancia del contexto pues: “(...) según lo que conozco en diferentes instituciones no siempre las competencias digitales pueden explicarse a partir de la voluntad del profesor(a)”.

Esta aportación formó parte de las recomendaciones sobre la investigación en general, entre las que destacan el analizar los relatos de vida tanto en el plano personal como contextual y procurar el carácter abierto de la entrevista a partir de la profundización en las narraciones.

Por último, se realizó un ejercicio piloto del guion de entrevista modificado en el que se confirmó el carácter abierto de los ítems para profundizar en las vivencias de los docentes. Preparativos como la consulta del currículum vitae de los profesores y la conversación informal sobre la investigación favorecieron el desarrollo de la entrevista.

\section{Discusión y conclusiones}

La estrategia de evaluación por juicio de expertos permitió reunir evidencias de validez del contenido del guion de entrevista en profundidad y, a partir de su análisis, tomar decisiones para la mejora del instrumento. La pertinencia e importancia de varios indicadores fueron confirmadas. A partir de la síntesis de su contenido y de la adición de ítems el instrumento adquirió mayor consistencia al estar más acotado, mejorar su fundamentación y reducir posibles sesgos en la etapa preparatoria de la investigación. El guion definitivo se compone de tres dimensiones, diez categorías y 40 indicadores, y puede consultarse en el anexo 1. Constatamos con este estudio la valiosa ayuda del juicio de expertos para la evaluación del diseño de instrumentos de corte cualitativo.

Además, los propósitos de cada dimensión obtuvieron más claridad. La primera de ellas, "Desarrollo profesional docente", tiene un carácter transversal y secundario pues es el hilo para relatar el proceso de aprendizaje en relación con la CDD. El foco central de la investigación es la dimensión "Evolución de la CDD” porque abarca el recorrido cronológico y narrativo en torno a la adquisición y el desarrollo de la CDD; en este sentido, sus indicadores son ayudas para evocar relatos y reflexiones del profesorado. La dimensión "Contexto: Educación superior y cultura digital" trata del contexto institucional y sociocultural de las experiencias del profesorado universitario, por lo que sitúa el desarrollo de la CDD.

Es importante señalar que un relato puede presentar uno o más aspectos de la CDD o de ítems del guion. Por ejemplo, el manejo de información digital está presente en varios de los ejes de la docencia universitaria y es probable que implique reflexiones sobre el uso responsable y ético de las TIC. Así, los relatos de vida de los docentes serán una oportunidad para caracterizar la CDD en sus distintas manifestaciones y en su complejidad.

En cuanto a la comprensión de esta competencia, surgieron varios puntos para el debate. En primer lugar, se sugirió integrar la transferencia del conocimiento o vinculación 
como otro eje fundamental de la labor docente. En segundo lugar, la programación informática como área de la CDD fue cuestionada; ésta podría resultar pertinente según el perfil y el campo disciplinar del profesorado. Tercero, las áreas pedagógica y ética resultaron difíciles de concretar; respecto del área ética, algunas propuestas la consideran un aspecto transversal a los ejes de la labor docente (Prendes et al., 2018; Rangel, 2016). Por último, fue llamativo el énfasis de algunos jueces en el perfil digital de los estudiantes debido a su influencia en el desarrollo de la CDD, observación que resulta congruente con el planteamiento del modelo DigCompEdu (Redecker, 2017). En consecuencia, se tendrán presentes estos puntos para el análisis de los relatos.

Finalmente, el guion anexo constituye un material que podría ser retomado por futuras investigaciones desde una perspectiva complementaria a la tendencia mayormente cuantitativa en el campo. Así mismo, puede ser un recurso de apoyo para proyectos de formación docente e innovación educativa a fin de profundizar en las vivencias y reflexiones del profesorado sobre el desafío de la integración de TIC a la Educación Superior.

\section{Agradecimientos}

Agradecemos la colaboración de los jueces expertos en esta línea de debate. La estancia de investigación cuenta con el apoyo del Programa de Becas en el Extranjero del Consejo Nacional de Ciencia y Tecnología (CONACYT), México.

\section{Referencias}

Adams Becker, S., Cummins, M., Davis, A., Freeman, A., Hall Giesinger, C., \& Ananthanarayanan, V. (2017). The NMC Horizon Report: 2017 Higher Education Edition. Texas: The New Media Consortium. Recuperado de: https://www.nmc. org/publication/nmc-horizon-report-2017-higher-education-edition/

Ala-Mutka, K. (2011). Mapping digital competence: towards a conceptual understanding. Sevilla: Joint Research Centre. Recuperado de: http://ftp.jrc.es/ EURdoc/JRC67075_TN.pdf

Bandalos, D. (2018). Validity. En Measurement Theory and Applications for the Social Sciences (pp. 254-297). Nueva York-London: The Guilford Press.

Bardin, L. (1996). Análisis de contenido. Madrid: Ediciones Akal.

Bolívar, A., Domingo, J., \& Fernández, M. (2001). La investigación biográfico-narrativa en educación: Enfoque y metodología. España: La Muralla.

Cabero, J., \& Llorente, M. C. (2013). La aplicación del juicio de experto como técnica de evaluación de las Tecnologías de Información y Comunicación (TIC). Revista de Tecnología de Información y Comunicación en Educación, 7(2), 11-22.

Carrera, F. X., \& Coiduras, J. L. (2012). Identificación de la competencia digital del profesor universitario: un estudio exploratorio en el ámbito de las Ciencias Sociales. REDU Revista de Docencia Universitaria, 10(2), 273-298. 
Cejas-León, R., \& Navío, A. (2016). El modelo TPACK competencial. Elaboración de un cuestionario para el profesorado universitario [CD]. En J. Gairín. (Ed.), Aprendizaje situado y aprendizaje conectado: Implicaciones para el trabajo. Madrid: Wolters Kluwers.

Davis, L. (1992). Instrument review: Getting the most from a panel of experts: Applied Nursing Research. Clinical Methods, 5(4), 194-197.

Delgado-Rico, E., Carretero-Dios, H., \& Ruch, W. (2012). Content validity evidences in test development: An applied perspective. International Journal of Clinical and Health Psychology, 12(3), 449-460.

Díaz-Barriga, A. (2011). Competencias en educación. Corrientes de pensamiento e implicaciones para el currículo y el trabajo en el aula. Revista Iberoamericana de Educación Superior (RIES), 5(2), 3-24.

Escobar-Pérez, J., \& Cuervo-Martínez, Á. (2008). Validez de contenido y juicio de expertos: una aproximación a su utilización. Avances en medición, 6(1), 27-36.

Fernández, M. (2006). Desarrollo profesional docente. Granada: Grupo Editorial Universitario.

Freelon, D. (2010). ReCal: Intercoder reliability calculation as a web service.International Journal of Internet Science, 5(1), 20-33.

Freelon, D, (2017). ReCal3: Reliability for 3+ Coders [en línea]. Recuperado de: http:// dfreelon.org/utils/recalfront/recal3/

Gallardo-Echenique, E., Minelli, J., Marqués-Molias, L., \& Esteve-Mon, F. (2015). Digital competence in Knowledge Society. Journal of Online Learning and Teaching, 11(1), $1-16$.

Hernández-Sampieri, R., Fernández, C., \& Baptista, P. (2014). Metodología de la investigación. Madrid: McGraw-Hill.

Hynes, S. N., Richard, D. C. S., \& Kubany, E. S. (1995). Content Validity in Psychological Assessment: A Functional Approach to Concepts and Methods. Psychological Assessment, 7(3), 238-247. doi: 1040-3590/95/\$3.00

Janssen, J., Stoyanov, S., Ferrari, A., Punie, Y., Pannekeet, K., \& Sloep, P. (2013). Experts' views on digital competence: Commonalities and differences. Computers \& Education, 68, 473-481. doi: 10.1016/j.compedu.2013.06.008

Kelchtermans, G. (1999). Narrative-Biographical Research on Teachers' Professional Development: Exemplifying a Methodological Research Procedure. En On the Threshold of the 21st Century: Challenges and Opportunities. Annual Meeting of the American Educational Research Association (AERA-symposium), (pp. 2-22). Montreal: $\mathrm{n} / \mathrm{d}$.

Lawshe, C. H. (1975). A quantitative approach to content validity. Personnel Psychology, 28(4), 563-575. doi: 10.1111/j.1744-6570.1975.tbo1393.x 
López de Ullibarri, I., \& Pita, S. (1999). Medidas de concordancia: el índice Kappa. Cadernos de atención primaria, 6(4), 223-226.

Martínez-Piñeiro, E., Vila, E., \& Grewerc, A. (2018). El papel de la familia en la construcción de la competencia digital. RISTI: Revista Ibérica de Sistemas e Tecnologias de Informação, (28), 2-13. doi: 10.17013/risti.28.1-13

Organización de las Naciones Unidas para la Educación, la Ciencia y la Cultura (2011). UNESCO ICT competency framework for teachers. París: UNESCO.

Padilla-Hernández, A. L., Gámiz-Sánchez, V. M., \& Romero-López, M. A. (2018). Selección de categorías para el estudio de la evolución de la competencia digital docente del profesorado en Educación Superior. RIITE Revista Interuniversitaria de Investigación en Tecnología Educativa, (4), 55-67. doi: 10.6018/riite/2018/327881

Pedrosa, I., Suárez-Álvarez, J., \& García-Cueto, E. (2013). Evidencias sobre la validez de contenido: avances teóricos y métodos para su estimulación. Acción Psicológica, 1O(2), 3-20. doi: 10.5944/ap.10.2.11820

Polit, D. F., Beck, C. T., \& Owen, S. V. (2007). Is the CVI an acceptable indicator of content validity?: Appraisal and recommendations. Research in Nursing and Health, 3O(4), 459-467. doi: 10.1002/nur.20199

Pozos, K. V. (2013). Digital competence of higher education teachers for the knowledge society: a preliminary approach of a need assessment study in mexican universities. En 6th International Conference of Education, Research and Innovation (ICERI 2013), (pp. 5533-5543). Sevilla: IATED.

Prendes, M. P. (2018). La Tecnología Educativa en la Pedagogía del siglo XXI: una visión en 3D. RIITE Revista Interuniversitaria de Investigación en Tecnología Educativa, (4), 6-16. doi: 10.6018/riite/2018/335131

Prendes, M. P., Martínez, F., \& Gutiérrez, I. (2018). Competencia digital: una necesidad del profesorado universitario en el siglo XXI. RED Revista de Educación a Distancia, año XVI (56), 1-22. doi: 10.6018/red/56/7

Rangel, A. (2016). Competencias docentes digitales. Propuesta de un perfil. Píxel-Bit. Revista de Medios y Educación, (46), 235-248. doi: 10.12795/pixelbit.2015.146.15

Redecker, C. (2017). European Framework for the Digital Competence of Educators: DigCompEdu. Sevilla: Joint Research Centre.

Rodrigues, L., Martins, M., Pinto, C.C., \& Teixeira, E. (2018). Tecnologia educacional para mediar práticas educativas sobre alimentação complementar na Amazônia: estudo de validação. RISTI: Revista Ibérica de Sistemas e Tecnologias de Informação, (28), 29-40. doi: 10.17013/risti.28.29-40

Sireci, S. G. (1998). The construct of content validity. Social Indicators Research, 45(13), 83-117.

Tejada, J., \& Pozos, K. V. (2018). Nuevos escenarios y competencias digitales docentes: Hacia la profesionalización docente con TIC. Profesorado, Revista de Currículum y Formación del Profesorado, 22(1), 41-67. 
Wynd, C., Schmidt, B., \& Atkins, M. (2003). Two quantitative approaches for estimating content validity. Western Journal of Nursing Research, 25(5), 508-518. doi: $10.1177 / 0193945903252998$

Zabalza, M. Á. (2003). Competencias docentes del profesorado universitario: calidad y desarrollo profesional. Madrid: Narcea.

\section{Anexo: Guion de entrevista en profundidad sobre la evolución de la CDD de profesores universitarios}

1. Desarrollo profesional docente

1.1. Trayectoria profesional

- Antecedentes relacionados con la docencia

- Razones para elegir la docencia como profesión

- Evolución de la carrera profesional: primera etapa, desarrollo y momento actual

1.2. Perfil profesional

- Autopercepción y motivaciones profesionales

- Perspectiva de futuro

2. Evolución de la competencia digital docente (CDD)

2.1. Adquisición y desarrollo de la CDD

- Antecedentes en relación con las TIC

- Experiencia en educación básica, profesional y universitaria relacionada con las TIC

- Primeras experiencias para la adquisición de la CDD

- Experiencias significativas para desarrollar la CDD

- Dificultades en relación con el desarrollo de la CDD

- Recursos digitales

- Influencia de ámbitos familiar, social y laboral

2.2. Los ejes de la docencia universitaria y áreas de la CDD

- Pedagógica

- Informacional

- Comunicativo-colaborativa

- Técnica

- Ejes: docencia, investigación, gestión, y transferencia de conocimiento o vinculación.

2.3. Aprendizaje continuo

- Estrategias para actualizar su CDD

- Formación continua sistematizada o formal

- Colaboración (docentes, estudiantes, comunidad)

- Proyectos de investigación y de innovación

- Vías para compartir su experiencia

- Necesidades de aprendizaje para la CDD

2.4. Reflexiones sobre su CDD

- Cambios en el perfil docente

- Beneficios asociados a su CDD

- Problemáticas en relación con el uso de TIC 
- Implicaciones éticas y uso responsable de TIC

- Salud, seguridad y prevención de riesgos

3. Contexto: Educación Superior y cultura digital

3.1. Perfil digital de los estudiantes universitarios

- Percepción de la competencia digital de los estudiantes universitarios

- Oportunidades de desarrollo de la competencia digital en la universidad

- Necesidades de aprendizaje en relación con la competencia digital

3.2. Competencia digital de los equipos docentes

- Coordinación docente

- Grupos de investigación

- Grupos de innovación

3.3. Valoración del contexto institucional

- Conocimiento y participación en la política educativa sobre TIC

- Apoyo y recursos técnicos en su universidad

- Percepción de la influencia de las TIC en la mejora educativa

- Desafíos para la alfabetización digital crítica en su universidad

3.4. Valoración del contexto socio-cultural

- Impacto de la cultura digital en la Educación Superior y viceversa

- Reflexiones sobre las TIC y la digitalización en general en la sociedad 